\title{
Maslov's Quantization Conditions for the Bound States of the Hydrogen Atom
}

\author{
Akira YOSHIOKA \\ Tokyo Metropolitan University \\ (Communicated by K. Ogiue)
}

\section{Introduction}

The purpose of this paper is to show that Maslov's quantization condition determines the eigenvalues of the Schrödinger operator of the hydrogen atom, the angular momentum operator and the Lenz operator, and also determines multiplicities of the eigenspaces for the hydrogen atom. Namely, we concern the eigenvalue problem of the following Schrödinger operator on $\boldsymbol{R}^{3}$ :

$$
\hat{H}\left(x, \frac{h}{i} \frac{\partial}{\partial x}\right)=-\frac{h^{2}}{2} \Delta-\frac{1}{|x|}, \quad|x|=\left(\sum_{k=1}^{3} x_{k}^{2}\right)^{1 / 2},
$$

where $h$ is a positive parameter and $\Delta$ is the 3-dimensional Laplacian.

Maslov [7], [8] introduces his index and the quantization condition for Lagrangian submanifolds and studies the "asymptotic solutions" of the eigenvalue problems in quantum mechanics (cf. [4]). On the contrary, in our case, we get exact values. Thus, as far as these systems are concerned, we need not to consider the operator theory to obtain the exact quantum mechanical conclusion. What we need is only classical mechanics, invariant Lagrangian submanifolds and Maslov's quantization conditions.

Associated with (1), we consider the following commuting system of operators $\left\{\hat{H}(x,(h / i)(\partial / \partial x)), \hat{l}_{1}(x,(h / i)(\partial / \partial x)), \hat{e}_{1}(x,(h / i)(\partial / \partial x))\right\}$, where $\hat{l}_{1}(x,(h / i)$ $(\partial / x))$ and $\hat{e}_{1}(x,(h / i)(\partial / \partial x))$ are the angular momentum operator and the Lenz operator, respectively, defined by

$$
\hat{l}_{1}\left(x, \frac{h}{i} \frac{\partial}{\partial x}\right)=x_{2} \hat{p}_{3}-x_{3} \hat{p}_{2},
$$

Received July 11, 1985 


$$
\begin{aligned}
& \hat{e}_{1}\left(x, \frac{h}{i} \frac{\partial}{\partial x}\right)=x_{2} \hat{p}_{1} \hat{p}_{2}+x_{8} \hat{p}_{1} \hat{p}_{8}-x_{1}\left(\hat{p}_{2}^{2}+\hat{p}_{3}^{2}\right)+\hat{p}_{1}+\frac{x_{1}}{|x|}, \\
& \hat{p}_{k}=\frac{h}{i} \frac{\partial}{\partial x_{k}}, \quad k=1,2,3 .
\end{aligned}
$$

We investigate eigenfunctions of $\hat{H}$, which are also eigenfunctions of $\hat{l}_{1}, \hat{e}_{1}$. Denote the corresponding Hamiltonian functions of $\hat{H}, \hat{l}_{1}$ and $\hat{e}_{1}$ by

$$
\begin{gathered}
H(x ; p)=\frac{1}{2}|p|^{2}-\frac{1}{|x|}, \\
l_{1}(x ; p)=x_{2} p_{8}-x_{3} p_{2}, \\
e_{1}(x ; p)=p_{1}\langle x, p\rangle-x_{1}|p|^{2}+\frac{x_{1}}{|x|},
\end{gathered}
$$

where $\langle x, p\rangle=\sum_{k=1}^{3} x_{k} p_{k}$.

Let us denote the level set of $\left(H, l_{1}, e_{1}\right)$ by

$$
\begin{aligned}
L\left(E, \bar{l}_{1}, \bar{e}_{1}\right)= & \left\{(x ; p) \in T^{*}\left(R^{3} \backslash\{0\}\right) \mid H(x ; p)=-E(E>0),\right. \\
& \left.l_{1}(x ; p)=\bar{l}_{1}, e_{1}(x ; p)=\bar{e}_{1}\right\} .
\end{aligned}
$$

REMARK. Since our concern is the bound states, we have only to consider the negative parameter $-E(E>0)$.

As a main result, we have the following:

THEOREM 1. $L\left(E, \bar{l}_{1}, \bar{e}_{1}\right)$ satifies Maslov's quantization condition if and only if

$$
\begin{gathered}
E=E_{n}=\frac{1}{2 n^{2} h^{2}}, \\
\bar{l}_{1}=\bar{l}_{1, m}=m h, \\
\bar{e}_{1}=\bar{e}_{1, n_{1}, n_{2}}=\frac{n_{1}-n_{2}}{n},
\end{gathered}
$$

where $n, n_{1}, n_{2}, m \in Z, n_{1}, n_{2} \geqq 0$, and $n=n_{1}+n_{2}+|m|+1$.

Notations are as above. Comparing above values with the eigenvalues of $\hat{H}, \hat{l}_{1}$, $\hat{e}_{1}$, we get the following (cf. [5], p. 119, 131).

THEOREM 2. (i) $E_{n}, \bar{l}_{1, m}$ and $\bar{e}_{1, n_{1}, n_{2}}$ are just equal to eigenvalues of $\hat{H}, \hat{l}_{1}$ and $\hat{e}_{1}$, respectively. 
(ii) Moreover, for each $E_{n}\left(=1 / 2 n^{2} h^{2}\right)$, the number of elements of

$$
\left\{L\left(E_{n}, \bar{l}_{1, m}, \bar{e}_{1, n_{1}, n_{2}}\right)\left|n=n_{1}+n_{2}+\right| m \mid+1, n_{1}, n_{2} \geqq 0\right\}
$$

is equal to the multiplicity of the eigenspace of $\hat{H}$ corresponding to $E_{n}$.

Using another commuting system, Leray [6] showed that the quantization condition gives exactly eigenvalues for the hydrogen atom with Zeeman effect. To be precise, he considered the following operator on $\boldsymbol{R}^{\mathbf{3}}$.

$$
\hat{H}_{1}\left(x, \frac{h}{i} \frac{\partial}{\partial x}\right)=\hat{H}\left(x, \frac{h}{i} \frac{\partial}{\partial x}\right)+\varepsilon \hat{l}_{1}\left(x, \frac{h}{i} \frac{\partial}{\partial x}\right),
$$

$\varepsilon \in \boldsymbol{R}$, where $\hat{H}$ and $\hat{l}_{1}$ are defined by (1) and (2), respectively. Associated with (3), he considered the commuting system $\left\{\hat{H}_{1}, \hat{l}_{1}, \sum_{k=1}^{3} \hat{l}_{k}^{2}\right\}$, where $\left(\hat{l}_{1}, \hat{l}_{2}, \hat{l}_{3}\right)=x \wedge(h / i)(\partial / \partial x)$, and corresponding Hamiltonian functions $H_{1}=H+$ $\varepsilon l_{1}, l_{1}, \sum_{k=1}^{3} l_{k}^{2}$, where $\left(l_{1}, l_{2}, l_{3}\right)=x \wedge p$. He constructed invariant Lagrangian submanifolds by the level set of $H_{1}, l_{1}$ and $\sum_{k=1}^{3} l_{k}^{2}$. By means of the quantization condition of these Lagrangian submanifolds, he obtained all eigenvalues of $\hat{H}_{1}$ and $\hat{l}_{1}$, (see Th. 4.2., Chap. III, $\S 1$, [6]).

Lastly, we remark that Leray [6] has given several examples of operators (i.e., Schrödinger, Klein-Gordon, Dirac operators) whose eigenvalues are exactly determined by the above classical method. We may expect a certain class of quantum mechanical models, of which the eigenvalue problems are exactly solved by the classical method.

Finally the author wishes to express his hearty thanks to Professor H. Omori for his encouragement.

\section{§1. Invariant Lagrangian submanifolds.}

Let $M$ be a symplectic manifold and $L$ be a subset of $M$. Suppose $F_{1}, F_{2}, \cdots, F_{l}$ are smooth functions on $M$. We call $L$ an invariant Lagrangian submanifold of $\left(F_{1}, F_{2}, \ldots, F_{l}\right)$ if $L$ satisfies the following two conditions; (i) $L$ is a Lagrangian submanifold of $M$, (ii) $L$ is invariant under $X_{H_{k}}, k=1,2, \cdots, l$, that is, $X_{H_{k}}(p) \in T_{p} L k=1,2, \cdots, l$ for every $p \in L$, where $X_{H_{k}}$ is a Hamiltonian vector field of $H_{k}$.

We set the Kepler manifold as

$$
U=\left\{(x ; p) \in T^{*}\left(\boldsymbol{R}^{3} \backslash\{0\}\right) \mid H(x ; p)<0\right\} .
$$

Remark that $L\left(E, \bar{l}_{1}, \bar{e}_{1}\right)$ is contained in $U$.

In this section, we prove the following.

Proposition 1.1. (a) For every point $(x ; p) \in U$, 


$$
\frac{1}{\sqrt{-2 H(x ; p)}} \geqq\left|l_{1}(x ; p)\right|+\frac{\left|e_{1}(x ; p)\right|}{\sqrt{-2 H(x ; p)}} .
$$

(b) If $(1 / \sqrt{2 E})>\left|\bar{l}_{1}\right|+\left(\left|\bar{e}_{1}\right| / \sqrt{2 E}\right)$, then $L\left(E, \bar{l}_{1}, \bar{e}_{1}\right)$ is an invariant Lagrangian submanifold of $\left(H, l_{1}, e_{1}\right)$.

(c) If $(1 / \sqrt{2 E})=\left|\bar{l}_{1}\right|+\left(\left|\bar{e}_{1}\right| / \sqrt{2 E}\right)$, then $L\left(E, \bar{l}_{1}, \bar{e}_{1}\right)$ is contained in two dimensional submanifold $\left(\right.$ for $\bar{l}_{1} \cdot \bar{e}_{1} \neq 0$ ), or one dimensional submanifold (for $\bar{l}_{1} \cdot \bar{e}_{1}=0$ ), respectively.

First of all, we consider the following theorem. Let $F_{1}, F_{2}, \cdots, F_{n}$ be smooth functions on $2 n$-dimensional symplectic manifold $M$. Suppose they are in involution, that is, $\left\{F_{j}, F_{k}\right\}=0(j, k=1,2, \cdots, n)$, where $\{$, is the Poisson bracket induced by the symplectic form. We denote the level set by

$$
M_{f}=\left\{x \in M \mid F_{k}(x)=f_{k}, k=1,2, \cdots, n\right\} .
$$

TheOREM A ([3], [1]). If $n$ functions $F_{k}$ are independent on $M_{f}$, i.e., 1-forms $d F_{k}$ are linearly independent at each point of $M_{f}$, then $M_{f}$ is invariant Lagrangian submanifold of $\left(F_{1}, F_{2}, \cdots, F_{n}\right)$. Furthermore, assume $M_{f}$ is compact, then it is diffeomorphic to n-torus.

Direct calculation yields the following.

LEMMA 1.2. $H, l_{1}$ and $e_{1}$ are in involution.

In view of theorem $A$ and Lemma 1.2, we have to study the independency of $H, l_{1}$ and $e_{1}$. Besides this, we also have to study the possible values of $H, l_{1}$ and $e_{1}$, since there exists some relation among them.

In what follows, we transfer the system to $T^{*} S^{s}$ through the Souriau's symplectic diffeomorphism and we investigate the above on $T^{*} S^{3}$.

We prepare notations.

$$
\begin{gathered}
S^{3}=\left\{y \in R^{4} \mid\|y\|^{2}=y_{1}^{2}+y_{2}^{2}+y_{3}^{2}+y_{4}^{2}=1\right\}, \\
T^{*} S^{3}=\left\{(y ; \xi) \in R^{4} \times R^{4} \mid y \in S^{3},\langle y, \xi\rangle=\sum_{k=1}^{4} y_{k} \xi_{k}=0\right\}, \\
\dot{T}^{*} S^{3}=T^{*} S^{3} \mid(0 \text {-section), } \\
C=\dot{T}_{q}^{*} S^{3}, \quad \text { where } \quad q=(0,0,0,1), \\
\omega=\sum_{k=1}^{4} \xi_{k} d y_{k} \mid \dot{T}^{*} S^{3} \quad \text { (canonical 1-form). }
\end{gathered}
$$


We define the diffeomorphism (See [9].)

$$
\psi: \stackrel{\circ}{*}^{*} S^{3} \backslash C \longrightarrow U
$$

by

$$
\left\{\begin{array}{l}
x=-\|\xi\|^{2}\left\{-\hat{\xi}_{4} y^{\prime}+y_{4} \hat{\xi}^{\prime}-\hat{\xi}^{\prime} \cos \zeta(y ; \xi)+y^{\prime} \sin \zeta(y ; \xi)\right\}, \\
p=\frac{\hat{\xi}^{\prime} \sin \zeta(y ; \xi)+y^{\prime} \cos \zeta(y ; \xi)}{\|\xi\|\left(\hat{\xi}_{4} \sin \zeta(y ; \xi)+y_{4} \cos \zeta(y ; \xi)-1\right)}
\end{array}\right.
$$

where $y=\left(y^{\prime}, y_{4}\right), \quad \hat{\xi}=\left(\hat{\xi}^{\prime}, \hat{\xi}_{4}\right), \quad \hat{\xi}_{k}=\xi_{k} /\|\xi\|, \quad k=1,2,3,4$, and $\zeta(y ; \xi)$ is the function satisfying the equation

$$
\zeta=-\hat{\xi}_{4} \cos \zeta+y_{4} \sin \zeta,
$$

Note that (1.2) has the unique solution $\zeta=\zeta(y ; \xi)$ and $\zeta(y ; \xi)$ is smooth on $\stackrel{\circ}{T}^{*} S^{3} \backslash C$ and continuous on $\stackrel{\circ}{T}^{*} S^{3}$.

Through $\psi$, the system can be written as follows on $\dot{T}^{*} S^{3}$.

Proposition 1.3 (cf. [9]).

$$
\psi^{*} \theta=\omega+d(2\|\xi\| \zeta(y ; \xi)),
$$

where $\theta=\sum_{k=1}^{3} p_{k} d x_{k}$.

Thus, $\psi$ is a symplectic diffeomorphism.

$$
\begin{gathered}
\psi^{*}(-1 /(2 H))=\|\xi\|^{2}, \\
\psi^{*} l_{1}=y_{2} \xi_{3}-y_{3} \xi_{2}, \\
\psi^{*}\left(e_{1} /(-2 H)^{1 / 2}\right)=y_{1} \xi_{4}-y_{4} \xi_{1} .
\end{gathered}
$$

We set $l_{1}^{*}(y ; \xi)=y_{2} \xi_{3}-y_{3} \xi_{2}, f_{1}^{*}(y ; \xi)=y_{1} \xi_{4}-y_{4} \xi_{1}$. In view of Proposition 1.3, we see the functions $\|\xi\|^{2}, l_{1}^{*}(y ; \xi)$ and $f_{1}^{*}(y ; \xi)$ are in involution.

For $a=\left(a_{1}, a_{2}, a_{3}\right)$, we denote the level set by

$$
T(a)=\left\{(y ; \xi) \in T^{*} S^{3} \mid\|\xi\|=a_{1}, l_{1}^{*}(y ; \xi)=a_{2}, f_{1}^{*}(y ; \xi)=a_{3}\right\} .
$$

Proposition 1.3 implies the following.

Corollary 1.4.

$$
L\left(E, \bar{l}_{1}, \bar{e}_{1}\right)=\psi(T(a) \backslash C),
$$

where $a=\left(a_{1}, a_{2}, a_{3}\right)$ satisfies

$$
a_{1}=\frac{1}{\sqrt{2 E}}, \quad a_{2}=\bar{l}_{1} \quad \text { and } \quad a_{3}=\frac{\bar{e}_{1}}{\sqrt{2 E}} .
$$


Now, we study the possible values and the independency of $\|\xi\|^{2}$, $l_{1}^{*}(y ; \xi)$ and $f_{1}^{*}(y ; \xi)$, so that we may get Proposition 1.1. Set

$$
\Gamma=\left\{(y ; \xi) \in \dot{T}^{*} S^{8} \mid\|\xi\|^{2}\left(y_{1}^{2}+y_{4}^{2}\right)=\xi_{1}^{2}+\xi_{4}^{2}, y_{1} \xi_{1}+y_{4} \xi_{4}=0\right\} \text {. }
$$

Then, we get the following.

LEMMA 1.5.

$$
\begin{aligned}
& \|\xi\| \geqq\left|l_{1}^{*}(y ; \xi)\right|+\left|f_{1}^{*}(y ; \xi)\right| \text { for every }(y ; \xi) \in \dot{T}^{*} S^{3} . \\
& \|\xi\|=\left|l_{1}^{*}(y ; \xi)\right|+\left|f_{1}^{*}(y ; \xi)\right| \quad \text { if and only if }(y ; \xi) \in \Gamma .
\end{aligned}
$$

Proof. By direct calculation, we have

$$
\begin{aligned}
& \left\{\|\xi\|^{2}\left(y_{1}^{2}+y_{4}^{2}\right)+\left(\xi_{1}^{2}+\xi_{4}^{2}\right)\right\}^{2}-4\|\xi\|^{2}\left|y_{1} \xi_{4}-y_{4} \xi_{1}\right|^{2} \\
& \quad=\left\{\|\xi\|^{2}\left(y_{1}^{2}+y_{4}^{2}\right)-\left(\xi_{1}^{2}+\xi_{4}^{2}\right)\right\}^{2}+4\|\xi\|^{2}\left(y_{1} \xi_{1}+y_{4} \xi_{4}\right)^{2} .
\end{aligned}
$$

Hence,

$$
\|\xi\|^{2}\left(y_{1}^{2}+y_{4}^{2}\right)+\left(\xi_{1}^{2}+\xi_{4}^{2}\right) \geqq 2\|\xi\|\left|y_{1} \xi_{4}-y_{4} \xi_{1}\right|,
$$

and the equality holds if and only if $(y ; \xi) \in \Gamma$.

Note that $\|\xi\| \geqq\left|l_{1}^{*}(y ; \xi)\right|$ and $\|\xi\| \geqq\left|f_{1}^{*}(y ; \xi)\right|$, since

$$
\|\xi\|^{2}=\sum_{j<k}\left(y_{j} \xi_{k}-y_{k} \xi_{j}\right)^{2} \text {. }
$$

An elementary computation yields

$$
\begin{aligned}
\left(\|\xi\|-\left|f_{1}^{*}(y ; \xi)\right|\right)^{2}-\left|l_{1}^{*}(y ; \xi)\right|^{2} & \\
\quad & =\|\xi\|^{2}\left(y_{1}^{2}+y_{4}^{2}\right)+\left(\xi_{1}^{2}+\xi_{4}^{2}\right)-2\|\xi\|\left|y_{1} \xi_{4}-y_{4} \xi_{1}\right| .
\end{aligned}
$$

Due to (1.4) and (1.5), we get the desired results.

Lemma 1.6. If $d\|\xi\|^{2}, d l_{1}^{*}(y ; \xi)$ and $d f_{1}^{*}(y ; \xi)$ are linearly dependent at $(\bar{y} ; \bar{\xi})$, then $(\bar{y} ; \bar{\xi})$ is contained in $\Gamma$.

Proof. We may assume $X_{\|\xi\|^{2}}, X_{l_{1}^{*}}$ and $X_{f_{1}^{*}}$ are linearly dependent at $(\bar{y} ; \bar{\xi})$, where $X_{\|\varepsilon\| \|^{2}}, X_{i_{1}^{*}}$ and $X_{f_{i}^{*}}$ are Hamiltonian vector fields of $\|\xi\|^{2}$, $l_{1}^{*}$ and $f_{1}^{*}$, respectively. Hence, there exists $(\alpha, \beta, \gamma)$ such that $(\alpha, \beta, \gamma) \neq$ $(0,0,0)$ and $\alpha X_{\left.|| \xi\right|^{2}}+\beta X_{l_{i}^{*}}+\gamma X_{f_{i}^{*}}=0 \cdots\left(^{*}\right)$.

$\left({ }^{*}\right)$ can be written as follows.

(i) In the case $\alpha=0$, we have

$$
\begin{array}{ll}
-\gamma \bar{y}_{4}=0, & -\gamma \bar{\xi}_{4}=0, \\
-\beta \bar{y}_{3}=0, & -\beta \bar{\xi}_{3}=0,
\end{array}
$$




$$
\begin{array}{ll}
\beta \bar{y}_{2}=0, & \beta \bar{\xi}_{2}=0, \\
\gamma \bar{y}_{1}=0, & \gamma \bar{\xi}_{1}=0,
\end{array}
$$

where $(\beta, \gamma) \neq(0,0)$. If $|\beta|=|\gamma|$, then we have $0=\gamma^{2}\left(\bar{y}_{1}^{2}+\bar{y}_{4}^{2}\right)+\beta^{2}\left(\bar{y}_{2}^{2}+\bar{y}_{3}^{2}\right)=\beta^{2}$, and this contradicts $(\beta, \gamma) \neq(0,0)$. If $|\gamma|>|\beta|$, then we have $0=\gamma^{2}\left(\bar{y}_{1}^{2}+\bar{y}_{4}^{2}\right)+$ $\beta^{2}\left(\bar{y}_{2}^{2}+\bar{y}_{3}^{2}\right)=\left(\gamma^{2}-\beta^{2}\right)\left(\bar{y}_{1}^{2}+\bar{y}_{4}^{2}\right)+\beta^{2}$. Thus, $\bar{y}_{1}=\bar{y}_{4}=\beta=0 \neq \gamma$, so that $\bar{\xi}_{1}=\bar{\xi}_{4}=0$. Hence, $(\bar{y} ; \bar{\xi}) \in \Gamma$. For $|\beta|>|\gamma|$, the same manner implies $(\bar{y} ; \bar{\xi}) \in \Gamma$.

(ii) In the case $\alpha \neq 0$. Set $\nu=\beta /(2 \alpha), \mu=\gamma /(2 \alpha)$. Then, $\left({ }^{*}\right)$ is written as

$$
\begin{array}{ll}
\bar{\xi}_{1}=\nu \bar{y}_{4}, & \bar{y}_{1}=-\frac{\nu}{\|\bar{\xi}\|^{2}} \bar{\xi}_{4}, \\
\bar{\xi}_{2}=\mu \bar{y}_{3}, & \bar{y}_{2}=-\frac{\mu}{\|\bar{\xi}\|^{2}}, \\
\bar{\xi}_{3}=-\mu \bar{y}_{2}, & \bar{y}_{3}=\frac{\mu}{\|\bar{\xi}\|^{2}} \bar{\xi}_{2}, \\
\bar{\xi}_{4}=-\nu \bar{y}_{1}, & \bar{y}_{4}=\frac{\nu}{\|\bar{\xi}\|^{2}} \bar{\xi}_{1} .
\end{array}
$$

Thus, $\bar{y}_{1}=\left(\nu^{2} /\|\bar{\xi}\|^{2}\right) \bar{y}_{1}$ and $\bar{y}_{4}=\left(\nu^{2} /\|\bar{\xi}\|^{2}\right) \bar{y}_{4}$. We get $\left(\bar{y}_{1}^{2}+\bar{y}_{4}^{2}\right)\left\{1-\left(\nu^{2} /\|\bar{\xi}\|^{2}\right)^{2}\right\}=0$. Hence, we have (i) $\bar{y}_{1}=\bar{y}_{4}=0$, or (ii) $\|\bar{\xi}\|^{2}=\nu^{2}$. If (i) holds, $\bar{\xi}_{1}=\bar{\xi}_{4}=0$. Thus $(\bar{y} ; \bar{\xi}) \in \Gamma$. If (ii) holds, then $\bar{\xi}_{1}^{2}+\bar{\xi}_{4}^{2}=\nu^{2}\left(\bar{y}_{1}^{2}+\bar{y}_{4}^{2}\right)=\|\bar{\xi}\|^{2}\left(\bar{y}_{1}^{2}+\bar{y}_{4}^{2}\right)$, and $\bar{y}_{1} \bar{\xi}_{1}+\bar{y}_{4} \bar{\xi}_{4}=$ $\nu \bar{y}_{1} \bar{y}_{4}-\nu \bar{y}_{4} \bar{y}_{1}=0$. Thus, $(\bar{y} ; \bar{\xi}) \in \Gamma$.

COROLLARY 1.7. If $a_{1}>\left|a_{2}\right|+\left|a_{3}\right|$, then $T(a)$ is an invariant Lagrangian Submanifold of $\left(\|\xi\|, l_{1}^{*}(y ; \xi), f_{1}^{*}(y ; \xi)\right)$, which is diffeomorphic to 3-torus.

Proof. On $T(a),\|\xi\|, l_{1}^{*}(y ; \xi)$ and $f_{1}^{*}(y ; \xi)$ are independent. Thus, theorem A gives the result.

On the other hand, for the case $a_{1}=\left|a_{2}\right|+\left|a_{3}\right|$, we have the following.

Set $\quad S S_{a_{2}}^{1}=\left\{\left(y_{2}, y_{3} ; \xi_{2}, \xi_{3}\right) \in \boldsymbol{R}^{2} \times \boldsymbol{R}^{2}\left|y_{2}^{2}+y_{3}^{2}=\right| a_{2}\left|/ a_{1}, \quad \xi_{2}^{2}+\xi_{3}^{2}=a_{1}\right| a_{2} \mid, \quad y_{2} \xi_{2}+\right.$ $\left.y_{3} \xi_{3}=0\right\}$ and

$$
S S_{a_{3}}^{1}=\left\{\left(y_{1}, y_{4} ; \xi_{1}, \xi_{4}\right) \in \boldsymbol{R}^{2} \times \boldsymbol{R}^{2}\left|y_{1}^{2}+y_{4}^{2}=\right| a_{3}\left|/ a_{1}, \xi_{1}^{2}+\xi_{4}^{2}=a_{1}\right| a_{3} \mid, y_{1} \xi_{1}+y_{4} \xi_{4}=0\right\} \text {. }
$$

We remark that $S S_{a_{k}}^{1}(k=1,2)$ is diffeomorphic to unit cosphere bundle of $S^{1}\left(a_{k} \neq 0\right)$, or the point $\left(a_{k}=0\right)$, respectively.

LEMMA 1.8. If $a_{1}=\left|a_{2}\right|+\left|a_{3}\right|$, then

$$
T(a) \subset S S_{a_{2}}^{1} \times S S_{a_{3}}^{1} .
$$


Proof. By means of Lemma 1.5 (b), $T(a) \subset \Gamma$. Thus, we have $a_{1}^{2}\left(y_{1}^{2}+y_{4}^{2}\right)=\xi_{1}^{2}+\xi_{4}^{2}, y_{1} \xi_{1}+y_{4} \xi_{4}=0, y_{1} \xi_{4}-y_{4} \xi_{1}=a_{3}$. We have

$$
a_{3}^{2}=\left(y_{1} \xi_{1}+y_{4} \xi_{4}\right)^{2}+\left(y_{1} \xi_{4}-y_{4} \xi_{1}\right)^{2}=\left(y_{1}^{2}+y_{4}^{2}\right)\left(\xi_{1}^{2}+\xi_{4}^{2}\right) \text {. }
$$

Hence, we get $y_{1}^{2}+y_{4}^{2}=\left|a_{3}\right| / a_{1}, \xi_{1}^{2}+\xi_{4}^{2}=a_{1}\left|a_{3}\right|$. On the other hand,

$$
\begin{gathered}
y_{2}^{2}+y_{3}^{2}=1-\left(y_{1}^{2}+y_{4}^{2}\right)=\left|a_{2}\right| / a_{1}, \\
\xi_{2}^{2}+\xi_{3}^{2}=a_{1}^{2}-\left(\xi_{1}^{2}+\xi_{4}^{2}\right)=a_{1}^{2}-a_{1}\left|a_{3}\right|=a_{1}\left|a_{2}\right|, \\
y_{2} \xi_{2}+y_{3} \xi_{3}=-\left(y_{1} \xi_{1}+y_{4} \xi_{4}\right)=0 .
\end{gathered}
$$

Thus, we get the desired result.

On account of Corollary 1.7 and Lemma 1.8, we have the following.

Proposition 1.9. (a) If $a_{1}>\left|a_{2}\right|+\left|a_{3}\right|$, then $T(a)$ is an invariant Lagrangian submanifold of $\left(\|\xi\|, l_{1}^{*}, f_{1}^{*}\right)$, which is diffeomorphic to 3-torus.

(b) If $a_{1}=\left|a_{2}\right|+\left|a_{3}\right|$, then $T(a)$ is contained in two dimensional submanifold (for $a_{2} \cdot a_{3} \neq 0$ ), or in one dimensional submanifold (for $a_{2} \cdot a_{3}=0$ ), respectively.

Now, we shall prove Proposition 1.1. Proposition 1.3 (b) and Lemma 1.5 (a) show Proposition 1.1 (a). Owing to Corollary 1.4 and Lemma 1.6, if $(1 / \sqrt{2 E})>\left|\bar{l}_{1}\right|+\left(\left|\bar{e}_{1}\right| / \sqrt{2 E}\right)$, then $H, l_{1}$ and $e_{1}$ are independent on $L\left(E, \bar{l}_{1}, \bar{e}_{1}\right)$. Thus, we get (b). If, $(1 / \sqrt{2 E})=\left|\bar{l}_{1}\right|+\left(\left|\bar{e}_{1}\right| / \sqrt{2 E}\right)$, then

$$
\psi^{-1}\left(L\left(E, \bar{l}_{1}, \bar{e}_{1}\right)\right) \subset T(a), \quad a_{1}=\left|a_{2}\right|+\left|a_{3}\right| \text {. }
$$

Hence, Proposion 1.9 (b) gives (c).

\section{§2. The Topology of $L\left(E, \bar{l}_{1}, \bar{e}_{1}\right)$ and action integrals.}

In this section, we consider the following.

(i) We investigate the topology of $T(a) \backslash C$, so that we know that of $L\left(E, \bar{l}_{1}, \bar{e}_{1}\right)$ (cf. Corollary 1.4).

(ii) We compute action integrals $\int_{0} \theta$ for the basis

$$
c \in H_{1}\left(L\left(E, \bar{l}_{1}, \bar{e}_{1}\right): Z\right)
$$

explicitly.

We begin by introducing a global parametrization into $T(a)$. Set $T^{3}=(\boldsymbol{R} / \boldsymbol{Z})^{3} \ni u=(\lambda, \mu, \nu)$, and $a=\left(a_{1}, a_{2}, a_{3}\right)$ where $a_{1}>\left|a_{2}\right|+\left|a_{3}\right|$. Remark that $T(a)$ is an invariant Lagrangian submanifold of $\left(\|\xi\|^{2}, l_{1}^{*}, f_{1}^{*}\right.$ ) (cf. Proposition 1.9). 
We define the mapping $\varphi_{a}: T^{3} \rightarrow \stackrel{T}{*}^{*} S^{3}$ by

$$
\begin{aligned}
& \varphi_{a}(u)=(y(u) ; \xi(u)) \\
& y_{1}(u)=-\frac{1}{2} M \cos (2 \lambda \pi)+\frac{1}{2} N \sin (2 \mu \pi), \\
& y_{2}(u)=\frac{1}{2} \tilde{M} \cos 2(\lambda+\mu+\nu) \pi-\frac{1}{2} \tilde{N} \sin (2 \nu \pi), \\
& y_{3}(u)=\frac{1}{2} \tilde{M} \sin 2(\lambda+\mu+\nu) \pi+\frac{1}{2} \tilde{N} \cos (2 \nu \pi), \\
& y_{4}(u)=-\frac{1}{2} M \sin (2 \lambda \pi)+\frac{1}{2} N \cos (2 \mu \pi), \\
& \xi_{1}(u)=a_{1}\left\{\frac{1}{2} M \sin (2 \lambda \pi)+\frac{1}{2} N \cos (2 \mu \pi)\right\}, \\
& \xi_{2}(u)=a_{1}\left\{-\frac{1}{2} \tilde{M} \sin 2(\lambda+\mu+\nu) \pi+\frac{1}{2} \tilde{N} \cos (2 \nu \pi)\right\}, \\
& \xi_{3}(u)=a_{1}\left\{\frac{1}{2} \tilde{M} \cos 2(\lambda+\mu+\nu) \pi+\frac{1}{2} \tilde{N} \sin (2 \nu \pi)\right\}, \\
& \xi_{4}(u)=a_{1}\left\{-\frac{1}{2} M \cos (2 \lambda \pi)-\frac{1}{2} N \sin (2 \mu \pi)\right\},
\end{aligned}
$$

where

$$
\begin{aligned}
& M=\left\{\left(a_{1}+a_{3}\right)^{2}-a_{2}^{2}\right\}^{1 / 2} / a_{1}, \\
& N=\left\{\left(a_{1}-a_{3}\right)^{2}-a_{2}^{2}\right\}^{1 / 2} / a_{1}, \\
& \tilde{M}=\left\{\left(a_{1}+a_{2}\right)^{2}-a_{3}^{2}\right\}^{1 / 2} / a_{1}, \\
& \tilde{N}=\left\{\left(a_{1}-a_{2}\right)^{2}-a_{3}^{2}\right\}^{1 / 2} / a_{1} .
\end{aligned}
$$

Proposition $2.1 \quad$ (a) $\varphi_{a}\left(T^{3}\right)=T(a)$.

(b) $\varphi_{a}$ is a diffeomorphism between $T^{3}$ and $T(a)$.

Proof. We denote the one parameter transformation groups of $X_{\|\varepsilon\|^{2}}$, $X_{l_{1}^{*}}$ and $X_{f_{1}^{*}}$ by $g_{1}^{t}, g_{2}^{t}$ and $g_{3}^{t}$, respectively. Since $X_{|| \xi||^{2}}, X_{l_{1}^{*}}$ and $X_{f_{1}^{*}}$ are commuting, so are $g_{k}^{t}$. Hence, $g\left(t_{1}, t_{2}, t_{3} ; p\right)=g_{1}^{t_{1}} \circ g_{2}^{t_{2}} \circ g_{3}^{t_{3}}(p)$ induces an additive $\boldsymbol{R}^{3}$ action on $\dot{T}^{*} S^{3}$. Set the point $p_{0}=(-M / 2, \tilde{M} / 2, \tilde{N} / 2, N / 2$; $\left.a_{1} N / 2, a_{1} \tilde{N} / 2, a_{1} \tilde{M} / 2,-a_{1} M / 2\right)$. Then, it is easily checked $p_{0} \in T(a)$. Put 
$\widetilde{g}_{a}\left(t_{1}, t_{2}, t_{3}\right)=g\left(t_{1}, t_{2}, t_{3} ; p_{0}\right) . \quad$ Then; $\tilde{g}_{a}: R^{3} \rightarrow T(a)$ is onto. $\quad \Gamma_{0}=\left\{\left(t_{1}, t_{2}, t_{3}\right) \in\right.$ $\left.\boldsymbol{R}^{8} \mid \widetilde{g}_{a}\left(t_{1}, t_{2}, t_{3}\right)=p_{0}\right\}$ (the pre-image of $p_{0}$ ) is a discrete subgroup of $\boldsymbol{R}^{3}$. Thus, $\widetilde{g}_{a}$ induces a diffeomorphism $g_{a} ; R^{3} / \Gamma_{0} \rightarrow T(a)$.

On the other hand, $\tilde{g}_{a}$ can be written explicitly in the following manner.

Set three matrices $U_{1}(t), U_{2}(t), U_{3}(t)$ as

$$
\begin{aligned}
& U_{1}(t)=\left[\begin{array}{ll}
\cos \left(2 a_{1} t\right) I_{4}, & \left(1 / a_{1}\right) \sin \left(2 a_{1} t\right) I_{4} \\
-a_{1} \sin \left(2 a_{1} t\right) I_{4}, & \cos \left(2 a_{1} t\right) I_{4}
\end{array}\right], \\
& U_{2}(t)=\left[\begin{array}{ll}
R_{2,8}(t), & 0 \\
0 & R_{2,8}(t)
\end{array}\right], \\
& U_{3}(t)=\left[\begin{array}{ll}
R_{1,4}(t), & 0 \\
0 & R_{1,4}(t)
\end{array}\right],
\end{aligned}
$$

where $I_{4}$ is a $4 \times 4$ identity matrix and

$$
R_{2,3}(t)=\left[\begin{array}{rrrr}
1 & & & 0 \\
& \cos t, & -\sin t & \\
& \sin t, & \cos t & \\
0 & & & 1
\end{array}\right]
$$

(rotation on $y_{2}, y_{3}$ plane),

$$
R_{1,4}(t)=\left[\begin{array}{rrrr}
\cos t & & & -\sin t \\
& 1 & 0 & \\
& 0 & 1 & \\
\sin t & & & \cos t
\end{array}\right]
$$

(rotation on $y_{1}, y_{4}$ plane).

We remark that $U_{1}(t), U_{2}(t)$ and $U_{3}(t)$ are phase flows of $\|\xi\|^{2}, l_{1}^{*}(y ; \xi)$ and $f_{1}^{*}(y ; \xi)$, respectively. Thus, we have $\widetilde{g}_{a}\left(t_{1}, t_{2}, t_{3}\right)=U_{1}\left(t_{1}\right) U_{2}\left(t_{2}\right) U_{3}\left(t_{3}\right) p_{0}$. By means of the above expressions, the direct computation gives that the generators of $\Gamma_{0}$ are $v_{1}=\left(\pi / 2 a_{1}, \pi, \pi\right), v_{2}=\left(\pi / 2 a_{1}, \pi,-\pi\right), v_{3}=(0,2 \pi, 0)$. Hence, $\boldsymbol{R}^{3} / \Gamma_{0}$ is diffeomorphic to 3 -torus and the diffeomorphism $\varphi^{\prime}: T^{3} \rightarrow$ $\boldsymbol{R}^{3} / \Gamma_{0}$ is given by $\tilde{t}=\lambda v_{1}+\mu v_{2}+\nu v_{3}\left(\bmod \Gamma_{0}\right)$, where $\tilde{t}=\left(t_{1}, t_{2}, t_{3}\right) \in \boldsymbol{R}^{3} / \Gamma_{0}$, $u=(\lambda, \mu, \nu) \in T^{3}$. Computing $\varphi_{a}=g_{a} \varphi^{\prime}$, we get (2.1). Thus, we obtain Proposition 2.1.

Using the global parametrization, we know the topology of $T(a) \backslash C$, where $a=\left(a_{1}, a_{2}, a_{3}\right), a_{1}>\left|a_{2}\right|+\left|a_{3}\right|$. 
LEMMA 2.2. (a) $T(a)$ intersects $C$ if only if $a_{2}=0$.

(b) $\varphi_{a}^{-1}\left(T\left(a_{1}, 0, a_{3}\right) \cap C\right)=\{(3 / 4,0, \nu)\}\left(\subset T^{3}\right)$.

Proof. The intersection of $T(a)$ and $C$ is given by $y_{4}(u)=$ $-(1 / 2) M \sin (2 \lambda \pi)+(1 / 2) N \cos (2 \mu \pi)=1$, Remark that $M+N \leqq 2$. Then, $y_{4}(u)=1$ is equivalent to $M+N=2$ and $\sin (2 \lambda \pi)=-1, \cos (2 \mu \pi)=1$, namely, $a_{2}=0, \lambda=3 / 4, \mu=0$.

We denote $\varphi_{a}^{-1}\left(T\left(a_{1}, 0, a_{3}\right) \cap C\right)$ by $C_{0}=\{(3 / 4,0, \nu)\}$. We remark here $\left[C_{0}\right]$ is a generator of $H_{1}\left(T^{3} ; Z\right)$.

Thus, we have the following.

Proposition 2.3. (a) $T(a) \backslash C$ is diffeomorphic to $T^{3}$ (for $a_{2} \neq 0$ ), or $T^{3} \backslash C_{0}$ (for $a_{2}=0$ ), respectively.

(b) Assume $(1 / \sqrt{2 E})>\left|\bar{l}_{1}\right|+\left(\left|\bar{e}_{1}\right| / \sqrt{2 E}\right) . \quad L\left(E, \bar{l}_{1}, \bar{e}_{1}\right)$ is diffeomorphic to $T^{s}\left(\right.$ for $\left.\bar{l}_{1} \neq 0\right)$, or $T^{3} \backslash C_{0}$ (for $\left.\bar{l}_{1}=0\right)$, respectively.

Remark that $T^{3} \backslash C_{0}=\left(T^{2} \backslash\right.$ point $) \times S^{1}$. Then, it is easily checked

$$
H_{1}\left(T^{8} \backslash C_{0} ; Z\right)=H_{1}\left(T^{3} ; Z\right) \text {. }
$$

As a corollary of Proposition 2.3, we obtain the following.

Corollary 2.4. $H_{1}\left(L\left(E, \bar{l}_{1}, \bar{e}_{1}\right) ; \boldsymbol{Z}\right)=\boldsymbol{Z} \oplus \boldsymbol{Z} \oplus \boldsymbol{Z}$.

Now, we compute action integrals $\int_{c} \theta$. Set three closed curves $c_{1}(t)$, $c_{2}(t), c_{3}(t)$ in $T^{3}$ as

$$
\begin{array}{cl}
c_{1}: \lambda=t, \quad \mu=\frac{1}{2}, \quad \nu=0, \\
c_{2}: \lambda=0, \quad \mu=t, \quad \nu=0, \\
c_{3}: \lambda=0, \quad \mu=\frac{1}{2}, \quad \nu=t, \\
0 \leqq t \leqq 1 .
\end{array}
$$

Note $c_{k} \quad k=1,2,3$ are contained in $T^{3} \backslash C_{0}$. Thus, [ $\left.c_{k}\right] k=1,2,3$ are generators of both $H_{1}\left(T^{3} \backslash C_{0}: Z\right)$ and $H_{1}\left(T^{3}: Z\right)$ (see (2.2)). Put $\widetilde{c}_{k}=\psi \circ \varphi_{a} c_{k}$ $(k=1,2,3)$, where

$$
a_{1}=\frac{1}{\sqrt{2 E}}, \quad a_{2}=\bar{l}_{1} \quad \text { and } \quad a_{3}=\frac{\bar{e}_{1}}{\sqrt{\overline{2 E}}},
$$

$(1 / \sqrt{2 E})>\left|\bar{l}_{1}\right|+\left(\left|\bar{e}_{1}\right| / \sqrt{2 E}\right)$. Then, we get generators $\left[\widetilde{c}_{k}\right] \in H_{1}\left(L\left(E, \bar{l}_{1}, \bar{e}_{1}\right): Z\right)$, $k=1,2,3$. 
Proposition 2.5.

$$
\begin{aligned}
& \int_{{\tilde{\tau_{1}}}_{1}} \theta=\pi\left(\frac{1}{\sqrt{2 E}}+\frac{\bar{e}_{1}}{\sqrt{2 E}}+\bar{l}_{1}\right), \\
& \int_{{\tilde{\tau_{2}}}_{2}} \theta=\pi\left(\frac{1}{\sqrt{2 E}}-\frac{\bar{e}_{1}}{\sqrt{2 E}}+\bar{l}_{1}\right), \\
& \int_{{\tilde{c_{3}}}_{3}} \theta=2 \pi \bar{l}_{1} .
\end{aligned}
$$

Proof. On account of Proposition 1.3 (a),

$$
\int_{\tilde{c_{k}}} \theta=\int_{\psi \varphi_{a} o_{k}} \theta=\int_{\varphi_{a} \sigma_{k}} \psi^{*} \theta=\int_{\varphi_{a} o_{k}} \omega=\int_{\sigma_{k}} \varphi_{a}^{*} \omega .
$$

Using (2.1), we have, for $\widetilde{c_{1}}$,

$$
\begin{aligned}
\int_{0_{1}} \varphi_{a}^{*} \omega & \left.=\int_{0}^{1} \varphi_{a}^{*} \omega\right\rfloor \dot{c}_{1}(t) \\
& =a_{1} \pi \int_{0}^{1}\left[\frac{M^{2}}{2}+\frac{\widetilde{M}^{2}}{2}-\frac{M N}{2} \sin (2 t \pi)-\frac{\widetilde{M} \tilde{N}}{2} \sin (2 t \pi)\right] d t \\
& =a_{1} \pi \frac{1}{2}\left(M^{2}+\widetilde{M}^{2}\right)=\pi\left(a_{1}+a_{2}+a_{8}\right) \\
& =\pi\left(\frac{1}{\sqrt{2 E}}+\frac{\bar{e}_{1}}{\sqrt{2 E}}+\bar{l}_{1}\right) .
\end{aligned}
$$

For $c_{2}, c_{3}$, the same manner gives the results.

§3. Maslov-indeces of $L\left(E, \bar{l}_{1}, \bar{e}_{1}\right)$.

In this section, we assume

$$
\frac{1}{\sqrt{2 E}}>\left|\bar{l}_{1}\right|+\frac{\left|\bar{e}_{1}\right|}{\sqrt{2 E}} \text { and } a_{1}>\left|a_{2}\right|+\left|a_{3}\right|
$$

(cf. Propositions 1.1 and 1.9).

We denote the Maslov-form of $L\left(E, \bar{l}_{1}, \bar{e}_{1}\right)$ by $m_{L}$. Our goal of this section is to prove the following.

Proposition 3.1. $\left\langle m_{L}, \widetilde{c_{1}}\right\rangle=\left\langle m_{L}, \widetilde{c_{2}}\right\rangle=2,\left\langle m_{L}, \widetilde{c_{3}}\right\rangle=0$.

First, we recall the definition of the Maslov-form briefly (cf. [2]). Let $\Lambda(n)$ be the Lagrangian Grassmannian manifold of $T^{*} R^{n}$, that is, the collection of Lagrangian subspaces. The unitary group $U(n)$ acts on $\Lambda(n)$ transitively and $\Lambda(n) \simeq U(n) / O(n)$. Let $\lambda_{I m}=\{(x, 0)\} \in \Lambda(n)$. Hence, for any 
$\lambda \in \Lambda(n)$, there exists $W \in U(n)$ such that $\lambda=W \cdot \lambda_{I m} . \quad$ Put $\operatorname{Det}^{2} \lambda=(\operatorname{det} W)^{2}$. Then, Det ${ }^{2}$ is the mapping of $\Lambda(n)$ to $S^{1}$. Let $M$ be a Lagrangian submanifold in $T^{*} R^{n}$. Then, $T_{q}^{*} M$ can be naturally identified with the Lagrangian subspace by translating $(q ; 0)$ to $(0 ; 0)$ in $T^{*} R^{n}$. Thus, we have the mapping $\tau: M \rightarrow \Lambda(n)$. The Maslov-form of $M$ is an element of $H^{1}(M ; \boldsymbol{Z})$ given by

$$
m=\left(\operatorname{Det}^{2} \circ \tau\right)^{*}\left(\frac{1}{2 \pi i} \frac{d z}{z}\right),
$$

where $z \in C,|z|=1$.

For Lagrangian submanifolds defined as level sets of functions, we have the following. Let $H_{1}(x ; p), H_{2}(x ; p), \cdots, H_{n}(x ; p)$ be smooth functions on the domain $D$ in $T^{*} R^{n}$. Suppose they are in involution. We denote their level set by

$$
M_{f}=\left\{(x ; p) \in D \mid H_{k}(x ; p)=f_{k}, k=1,2, \cdots, n\right\} .
$$

Assume $M_{f}$ be an invariant Lagrangian submanifold of $\left(H_{1}, H_{2}, \cdots, H_{n}\right)$.

Proposition 3.2. The Maslov-form $m$ of $M_{f}$ can be written as $m=(1 / \pi) d \operatorname{Arg} \operatorname{det}\left(H_{p}+i H_{x}\right)$,

where

$$
H_{p}=\left(\frac{\partial H_{k}}{\partial p_{j}}\right), \quad H_{x}=\left(\frac{\partial H_{k}}{\partial x_{j}}\right), \quad j, k=1,2, \cdots, n .
$$

Proof. Suppose $q=(x ; p) \in M_{f}$ can be parametrized by $x$-variables. Let $S(x)$ be the generating function of $M_{f}$ near $q$. Then, we have $H_{k}\left(x ; S_{x}(x)\right)=f_{k}, k=1,2, \cdots, n$ and $\tau(q)=\left\{\left(X ; S_{x x}(x) \cdot X\right) \mid X \in \boldsymbol{R}^{n}\right\}$. Therefore,

$$
\left(\operatorname{Det}^{2} \circ \tau\right)(q)=\operatorname{det} \frac{E-i S_{x x}(x)}{E+i S_{x x}(x)} \quad \text { (cf. [2] Cor. 3.4.3). }
$$

On the other hand, $S_{x x}(x)=-H_{x} \cdot H_{p}^{-1}$. Hence, substituting these into (3.1), we get the desired form. Regarding other parametrizations, the above method, combined with the Legendre transformation, gives the result.

In what follows, we will compute Maslov-indeces for $L\left(E, \bar{l}_{1}, \bar{e}_{1}\right)$. To this end, we introduce another global parametrization into $T(a)$, which is convenient to compute Maslov-indeces.

Consider the function $\varphi_{a}^{*} \zeta$ on $T^{3}$. In view of (1.2) and (2.1), it is easily checked $\varphi_{a}^{*} \zeta$ satisfies the following equation:

For any $(\lambda, \mu) \in \boldsymbol{R}^{2}$,

$$
\tilde{\zeta}=\frac{1}{2} M \cos (2 \lambda \pi+\tilde{\zeta})+\frac{1}{2} N \sin (2 \mu \pi+\tilde{\zeta}) .
$$


Remark that (3.2) has the unique solution $\tilde{\zeta}=\varphi_{a}^{*} \zeta$. We denote this by $\zeta_{a}(\lambda, \mu)$. Note that $\zeta_{a}(\lambda, \mu)$ is smooth in the case $a_{2} \neq 0$, and continuous in the case $a_{2}=0$.

Define the map $\tilde{\boldsymbol{\kappa}}_{a}: \boldsymbol{R}^{3} \longrightarrow \boldsymbol{R}^{3} \quad$ by

$$
\begin{aligned}
& (\lambda, \mu, \nu) \longmapsto\left(s_{1}, s_{2}, \nu\right) \\
& \left\{\begin{array}{l}
s_{1}(\lambda, \mu, \nu)=\lambda+\frac{1}{2 \pi} \zeta_{a}(\lambda, \mu), \\
s_{2}(\lambda, \mu, \nu)=\mu+\frac{1}{2 \pi} \zeta_{a}(\lambda, \mu), \\
\nu=\nu .
\end{array}\right.
\end{aligned}
$$

LEMMA 3.3. For every $\left(s_{1}, s_{2}\right) \in R^{2}$, the following equation has the unique solution $(\lambda, \mu) \in \boldsymbol{R}^{2}$.

$$
\left\{\begin{array}{l}
s_{1}=\lambda+\frac{1}{2 \pi} \zeta_{a}(\lambda, \mu), \\
s_{2}=\mu+\frac{1}{2 \pi} \zeta_{a}(\lambda, \mu) .
\end{array}\right.
$$

Proof. Set

$$
\rho_{a}\left(s_{1}, s_{2}\right)=\frac{1}{2} M \cos \left(2 s_{1} \pi\right)+\frac{1}{2} N \sin \left(2 s_{2} \pi\right),
$$

and $\bar{\lambda}=s_{1}-(1 / 2 \pi) \rho_{a}\left(s_{1}, s_{2}\right), \bar{\mu}=s_{2}-(1 / 2 \pi) \rho_{a}\left(s_{1}, s_{2}\right)$. Hence, it holds that

$$
\begin{aligned}
\rho_{a}\left(s_{1}, s_{2}\right)= & \frac{1}{2} M \cos \left(2 \bar{\lambda} \pi+\rho_{a}\left(s_{1}, s_{2}\right)\right) \\
& +\frac{1}{2} N \sin \left(2 \bar{\mu} \pi+\rho_{a}\left(s_{1}, s_{2}\right)\right) .
\end{aligned}
$$

The uniqueness of (3.2) shows $\rho_{a}\left(s_{1}, s_{2}\right)=\zeta_{a}(\bar{\lambda}, \bar{\mu})$. Thus, $(\bar{\lambda}, \bar{\mu})$ is a solution to $(\mathrm{E})$. Uniqueness is easy to see.

Set the map

$$
\begin{gathered}
\tilde{\tau}_{a}: R^{3} \longrightarrow R^{3} \quad \text { by } \\
\quad\left(s_{1}, s_{2}, \nu\right) \longmapsto(\lambda, \mu, \nu) \\
\begin{array}{l}
\lambda=s_{1}-\frac{1}{2 \pi} \rho_{a}\left(s_{1}, s_{2}\right), \\
\mu=s_{2}-\frac{1}{2 \pi} \rho_{a}\left(s_{1}, s_{2}\right), \\
\nu=\nu,
\end{array}
\end{gathered}
$$


where

$$
\rho_{a}\left(s_{1}, s_{2}\right)=\frac{1}{2} M \cos \left(2 s_{1} \pi\right)+\frac{1}{2} N \sin \left(2 s_{2} \pi\right)
$$

By means of the proof of Lemma 3.3, we have $\tilde{\tau}_{a}=\tilde{\kappa}_{a}^{-1}$. It is easily checked $\tilde{\kappa}_{a}\left(u+f_{k}\right)=\tilde{\kappa}_{a}(u)+f_{k}, k=1,2,3$, where $u=(\lambda, \mu, \nu), f_{1}=(1,0,0)$, $f_{2}=(0,1,0), f_{3}=(0,0,1)$. So, $\tilde{\kappa}_{a}$ and $\tilde{\tau}_{a}$ induce the maps

$$
\kappa_{a}: T^{3} \longrightarrow T^{3}, \tau_{a}: T^{3} \longrightarrow T^{3} .
$$

Thus, we get the following.

Proposition 3.4. $\kappa_{a}: T^{3} \rightarrow T^{3}$ is a homeomorphism and the inverse is $\tau_{a}$, which is given by (3.4).

By means of $\tau_{a}$, we get another global parametrization of $T(a)$ such that

$$
\begin{aligned}
\varphi_{a} \circ \tau_{a}: T^{3} & \longrightarrow T(a) \\
\left(s_{1}, s_{2}, \nu\right) & \longmapsto\left(y\left(s_{1}, s_{2}, \nu\right) ; \xi\left(s_{1}, s_{2}, \nu\right)\right) .
\end{aligned}
$$

COROLlary 3.5. For every $a=\left(a_{1}, a_{2}, a_{3}\right)$,

$$
\tau_{a}^{-1}\left(C_{0}\right)=\left\{\left(s_{1}, s_{2}, \nu\right) \mid s_{1}=\frac{3}{4}, s_{2}=0,(\bmod Z)\right\} .
$$

Now, using parameters $\left(s_{1}, s_{2}, \nu\right)$, we set other representatives of $\left[c_{k}\right] \in H_{1}\left(L\left(E, \bar{l}_{1}, \bar{e}_{1}\right): Z\right)$ and write the Maslov-form explicitly. Set three closed curves on $T^{3}$ as

$$
\begin{aligned}
& c_{1}^{\prime}: s_{1}=t, \quad s_{2}=\frac{1}{2}, \quad \nu=0, \\
& c_{2}^{\prime}: s_{1}=\frac{1}{4}, \quad s_{2}=t, \quad \nu=0, \\
& c_{3}^{\prime}: s_{1}=s_{2}=\frac{1}{2}, \quad \nu=t, \quad(0 \leqq t \leqq 1) .
\end{aligned}
$$

Corollary 3.5 shows $\tau_{a}\left(c_{k}^{\prime}\right), k=1,2,3$ are contained in $T^{3} \backslash C_{0}$.

LEMMA 3.6. $\left[\tau_{a}\left(c_{k}^{\prime}\right)\right]=\left[c_{k}\right] \in H_{1}\left(T^{3} \backslash C_{0}: Z\right), k=1,2,3$.

PROOF. $\tau_{a}\left(c_{1}^{\prime}\right)$ is written as

$$
\lambda(t)=t-\frac{1}{2} \rho_{a}\left(t, \frac{1}{2}\right),
$$




$$
\begin{aligned}
& \mu(t)=\frac{1}{2}-\frac{1}{2} \rho_{a}\left(t, \frac{1}{2}\right), \\
& \nu=0 .
\end{aligned}
$$

Since $\rho_{a}(0,(1 / 2))=\rho_{a}(1,(1 / 2)), \quad \tau_{a}\left(c_{1}^{\prime}\right) \sim c_{1}$ (homotopic). Thus, we have $\left[\tau_{a}\left(c_{1}^{\prime}\right)\right]=\left[c_{1}\right]$. About $c_{2}^{\prime}$ and $c_{2}$, the same method gives the result. For $c_{3}^{\prime}, c_{3}$, it is obvious.

\section{LEMMA 3.7.}

$$
\begin{gathered}
\left(\psi \circ \varphi_{a} \circ \tau_{a}\right)^{*} m_{L}=\frac{1}{\pi} d \operatorname{Arg}(R+i I), \\
R=-a_{1}^{2} M N \cos \left(2 s_{1} \pi\right) \sin \left(2 s_{2} \pi\right) \\
+\frac{1}{a_{1}^{4}(1-z)^{2}}\left(A_{1}^{2}+z^{2}\right) \\
-\frac{1}{a_{1}^{4}(1-z)^{4}}\left\{\left(\frac{a_{3}}{a_{1}}+A_{2}\right)^{2}+\rho_{a}^{2}(1-z)+\rho_{a}\left(\frac{a_{3}}{a_{1}}+A_{2}\right) A_{1}\right\}, \\
I=\left\{2+\frac{1}{a_{1}^{6}(1-z)^{3}}\right\} \frac{1}{a_{1}(1-z)^{2}} \\
\times\left\{-\left(\frac{a_{3}}{a_{1}}+A_{2}\right) A_{1}+\rho_{a}\left(z-z^{2}-A_{1}^{2}\right)\right\},
\end{gathered}
$$

where

$$
\begin{aligned}
& A_{1}=-\frac{1}{2} M \cos \left(2 s_{1} \pi\right)+\frac{1}{2} N \sin \left(2 s_{2} \pi\right), \\
& A_{2}=\frac{1}{2} M \sin \left(2 s_{1} \pi\right)+\frac{1}{2} N \cos \left(2 s_{2} \pi\right), \\
& z=-\frac{1}{2} M \sin \left(2 s_{1} \pi\right)+\frac{1}{2} N \cos \left(2 s_{2} \pi\right), \\
& \rho_{a}=\frac{1}{2} M \cos \left(2 s_{1} \pi\right)+\frac{1}{2} N \sin \left(2 s_{2} \pi\right) .
\end{aligned}
$$

Proof. Applying Proposition 3.2 to $L\left(E, \bar{l}_{1}, \bar{e}_{1}\right)$, we have

$$
m_{L}=\frac{1}{\pi} d \operatorname{Arg}(\widetilde{R}+i \widetilde{I}),
$$

where 


$$
\begin{gathered}
\widetilde{R}=p_{1}^{2} r^{2}-2 E\left(|p|^{2}-p_{1}^{2}\right)+\frac{1}{r}\left(\frac{1}{r}-\frac{x_{1}^{2}}{r^{3}}\right)-\langle x, p\rangle^{2} \\
-\frac{\langle x, p\rangle^{2}}{r^{3}}-\frac{x_{1} p_{1}\langle x, p\rangle}{r^{3}}, \\
\widetilde{I}=\left(2+\frac{1}{r^{3}}\right)\left\{\langle x, p\rangle|p|^{2}-\langle x, p\rangle p_{1}^{2}-\frac{\langle x, p\rangle}{r}+\frac{x_{1} p_{1}}{r}\right\}, \\
r=|x| .
\end{gathered}
$$

Owing to (1.1), we have

$$
\begin{gathered}
\psi^{*}\langle x, p\rangle=\frac{1}{\sqrt{2 E}} \zeta(y ; \xi), \\
\psi^{*} r=\frac{1}{2 E}\left(1-z^{\prime}(y ; \xi)\right), \\
\psi^{*}|p|^{2}=2 E \frac{1+z^{\prime}(y ; \xi)}{1-z^{\prime}(y ; \xi)}, \\
\psi^{*}\left(x_{1} p_{1}\right)=-\frac{1}{\sqrt{2 E}} \frac{\left\{\bar{e}_{1}+\left(\hat{\xi}_{1} \cos \zeta(y ; \xi)-y_{1} \sin \zeta(y ; \xi)\right)\right\}}{1-z^{\prime}(y ; \xi)} \\
\times\left(\hat{\xi}_{1} \sin \zeta(y ; \xi)+y_{1} \cos \zeta(y ; \xi)\right), \\
\psi^{*} p_{1}=-\sqrt{2 E} \frac{\hat{\xi}_{1} \sin \zeta(y ; \xi)+y_{1} \cos \zeta(y ; \xi)}{1-z^{\prime}(y ; \xi)}, \\
\psi^{*} x_{1}=\frac{1}{2 E}\left\{\bar{e}_{1}+\left(\hat{\xi}_{1} \cos \zeta(y ; \xi)-y_{1} \sin \zeta(y ; \xi)\right)\right\},
\end{gathered}
$$

where

$$
\begin{gathered}
\zeta(y ; \xi)=-\hat{\xi}_{4} \cos \zeta(y ; \xi)+y_{4} \sin \zeta(y ; \xi), \\
z^{\prime}(y ; \xi)=\hat{\xi}_{4} \sin \zeta(y ; \xi)+y_{4} \cos \zeta(y ; \xi) .
\end{gathered}
$$

On account of (2.1) and (3.4), we get

$$
\begin{aligned}
& \left(\varphi_{a} \circ \tau_{a}\right)^{*} \zeta=\rho_{a}\left(s_{1}, s_{2}\right), \\
& \left(\varphi_{a} \circ \tau_{a}\right)^{*} z^{\prime}=z\left(s_{1}, s_{2}\right), \\
& \left(\varphi_{a} \circ \tau_{a}\right)^{*}\left(\hat{\xi}_{1} \sin \zeta+y_{1} \cos \zeta\right)=A_{1}, \\
& \left(\varphi_{a} \circ \tau_{a}\right) *\left(\hat{\xi}_{1} \cos \zeta-y_{1} \sin \zeta\right)=A_{2},
\end{aligned}
$$




$$
\frac{1}{\sqrt{2 E}}=a_{1}, \quad \bar{e}_{1}=\frac{a_{8}}{a_{1}} .
$$

The above equalities yield

$$
\left(\psi \circ \varphi_{a} \circ \tau_{a}\right)^{*} \widetilde{R}=R \text { and }\left(\psi \circ \varphi_{a} \circ \tau_{a}\right) * \widetilde{I}=I \text {. }
$$

For the constants given in (2.1), the following inequalities hold.

LEMMA 3.8.

1) $1+\frac{1}{2}(M-N)>0,1+\frac{1}{2}(N-M)>0$.

2) $1+N-\frac{a_{3}}{a_{1}}>0,1+M+\frac{a_{3}}{a_{1}}>0$.

3) $0<\frac{1+\frac{1}{2} N-\sqrt{1+N-a_{3} / a_{1}}}{(1 / 2) M}<1$,

$$
0<\frac{1+\frac{1}{2} M-\sqrt{1+M+a_{3} / a_{1}}}{(1 / 2) N}<1 .
$$

Now, we are in a position to prove Proposition 3.1. On account of Lemma 3.6,

$$
\begin{gathered}
\left\langle m_{L}, \tilde{c}_{k}\right\rangle=\left\langle m_{L}, \psi \circ \varphi_{a} c_{k}\right\rangle=\left\langle m_{L}, \psi \circ \varphi_{a} \circ \tau_{a} c_{k}^{\prime}\right\rangle \\
=\left\langle\left(\psi \circ \varphi_{a} \circ \tau_{a}\right)^{*} m_{L}, c_{k}^{\prime}\right\rangle, \quad k=1,2,3 .
\end{gathered}
$$

On $c_{1}^{\prime}, R$ and $I$ are written as follows.

$$
\begin{aligned}
R=\frac{1}{a_{1}^{4}(1-z)^{2}} & \left(A_{1}^{2}+z^{2}\right) \\
-\frac{1}{a_{1}^{4}(1-z)^{4}} & \left\{\left(\frac{a_{3}}{a_{1}}+A_{2}\right)^{2}+\rho_{a}^{2}(1-z)+\rho_{a}\left(\frac{a_{3}}{a_{1}}+A_{2}\right) A_{1}\right\}, \\
I= & \left\{2+\frac{1}{a_{1}^{6}(1-z)^{8}}\right\} \frac{1}{a_{1}(1-z)^{2}} \\
& \times\left\{-\left(\frac{a_{3}}{a_{1}}+A_{2}\right) A_{1}+\rho_{a}\left(z-z^{2}-A_{1}^{2}\right)\right\},
\end{aligned}
$$

where

$$
A_{1}=-\frac{1}{2} M \cos (2 t \pi),
$$




$$
\begin{aligned}
& A_{2}=\frac{1}{2} M \sin (2 t \pi)-\frac{1}{2} N, \\
& z=-\frac{1}{2} M \sin (2 t \pi)-\frac{1}{2} N, \\
& \rho_{a}=\frac{1}{2} M \cos (2 t \pi) .
\end{aligned}
$$

Thus, we have $A_{1}=-\rho_{a}$ and $A_{2}=-z-N$. Putting these into $R$ and $I$, we have

$$
\begin{gathered}
R=\frac{1}{a_{1}^{4}(1-z)^{4}}\left(\rho_{a}^{2}+z^{2}+N-\frac{a_{3}}{a_{1}}\right)\left\{(1-z)^{2}-\left(1+N-\frac{a_{3}}{a_{1}}\right)\right\}, \\
I=-\left\{2+\frac{1}{a_{1}^{6}(1-z)^{3}}\right\} \frac{1}{a_{1}(1-z)^{2}}\left\{\rho_{a}^{2}+z^{2}+N-\frac{a_{3}}{a_{1}}\right\} \rho_{a} .
\end{gathered}
$$

By means of Lemma 3.8,

$$
\begin{gathered}
1-z=1+\frac{1}{2} N+\frac{1}{2} M \sin (2 t \pi) \geqq 1+\frac{1}{2}(N-M)>0, \\
\rho_{a}^{2}+z^{2}+N-\frac{a_{3}}{a_{1}}=\frac{1}{2} N^{2}+N+\frac{1}{2} M N \sin (2 t \pi) \\
\geqq N\left\{1+\frac{1}{2}(N-M)\right\}>0 . \\
(1-z)^{2}-\left(1+N-\frac{a_{3}}{a_{1}}\right) \\
=\left(1-z+\sqrt{1+N-a_{3} / a_{1}}\right)(M / 2) \\
\quad \times\left\{\sin (2 t \pi)+\frac{1+\frac{1}{2} N-\sqrt{1+N-a_{3} / a_{1}}}{M / 2}\right\} .
\end{gathered}
$$

Hence, we have

$R=$ (positive function)

$$
\times\left\{\sin (2 t \pi)+\frac{1+\frac{1}{2} N-\sqrt{1+N-a_{3} / a_{1}}}{M / 2}\right\},
$$

$I=($ negative function $) \times \cos (2 t \pi)$.

Remark $0<\left(1+(1 / 2) N-\sqrt{1+N-a_{3} / a_{1}}\right) / M / 2<1$. We see $\operatorname{Arg}(R+i I)$ varies 
434

$2 \pi$ for $0 \leqq t \leqq 1$. Thus, we get $\left\langle m_{L}, \widetilde{c}_{1}\right\rangle=2$. On $c_{2}^{\prime}$, we have

$$
\begin{aligned}
& A_{1}=\frac{1}{2} N \sin (2 t \pi), \\
& A_{2}=\frac{1}{2} M+\frac{1}{2} N \cos (2 t \pi), \\
& z=-\frac{1}{2} M+\frac{1}{2} N \cos (2 t \pi), \\
& \rho_{a}=\frac{1}{2} N \sin (2 t \pi), \\
& A_{1}=\rho_{a}, \quad A_{2}=z+M .
\end{aligned}
$$

Hence, we get

$$
\begin{aligned}
R= & \frac{1}{a_{1}^{4}(1-z)^{4}}\left(\rho_{a}^{2}+z^{2}+M+\frac{a_{3}}{a_{1}}\right)\left\{(1-z)^{2}-\left(1+M+\frac{a_{8}}{a_{1}}\right)\right\}, \\
I= & -\left\{2+\frac{1}{a_{1}^{6}(1-z)^{8}}\right\} \frac{1}{a_{1}(1-z)^{2}} \\
& \times\left(\rho_{a}^{2}+z^{2}+M+\frac{a_{8}}{a_{1}}\right) \rho_{a} .
\end{aligned}
$$

Lemma 3.8 gives

$$
\begin{aligned}
& \begin{aligned}
& 1-z=1+\frac{1}{2} M- \frac{1}{2} N \cos (2 t \pi)>0, \\
& \rho_{a}^{2}+z^{2}+M+\frac{a_{8}}{a_{1}}=\frac{1}{2} M^{2}+M-\frac{1}{2} M N \cos (2 t \pi) \\
& \geqq\left.M 1+\frac{1}{2}(M-N)\right\}>0, \\
&(1-z)^{2}-\left(1+M+\frac{a_{8}}{a_{1}}\right)=\left(1-z+\sqrt{1+M+a_{3} / a_{1}}\right)(N / 2) \\
& \quad \times\left\{-\cos (2 t \pi)+\frac{1+\frac{1}{2} M-\sqrt{1+M+a_{3} / a_{1}}}{N / 2}\right\} .
\end{aligned}
\end{aligned}
$$

Thus, we obtain

$$
R=\text { (positive function) }
$$




$$
\begin{aligned}
& \quad\left\{-\cos (2 t \pi)+\frac{1+\frac{1}{2} M-\sqrt{1+M+a_{3} / a_{1}}}{N / 2}\right\}, \\
& I=(\text { negative function }) \times \sin (2 t \pi) .
\end{aligned}
$$

Hence, $\left\langle m_{L}, \widetilde{c}_{2}\right\rangle=2$.

Since $R$ and $I$ exclude $\nu$ variable, $\operatorname{Arg}(R+i I)$ is constant on $c_{3}^{\prime}$. Thus, $\left\langle m_{L}, \widetilde{c}_{3}\right\rangle=0$. Hence, we get Proposition 3.1.

\section{§4. Proof of theorems.}

For $L\left(E, \bar{l}_{1}, \bar{e}_{1}\right),(1 / \sqrt{2 E})>\left|\bar{l}_{1}\right|+\left(\left|\bar{e}_{1}\right| / \sqrt{2 E}\right)$, the quantization condition is as follows.

$$
\frac{1}{h} \int_{\tilde{c}_{k}} \theta-\frac{\pi}{2}\left\langle m_{L}, \widetilde{c}_{k}\right\rangle=2 \pi n_{k}, \quad n_{k} \in Z, \quad k=1,2,3 .
$$

By means of Propositions 2.5 and 3.1, we have

$$
\begin{aligned}
& \frac{\pi}{h}\left(\frac{1}{\sqrt{2 E}}+\frac{\bar{e}_{1}}{\sqrt{2 E}}+\bar{l}_{1}\right)-\frac{\pi}{2} \times 2=2 \pi \widetilde{n}_{1}, \\
& \frac{\pi}{h}\left(\frac{1}{\sqrt{2 E}}-\frac{\bar{e}_{1}}{\sqrt{2 E}}+\bar{l}_{1}\right)-\frac{\pi}{2} \times 2=2 \pi \widetilde{n}_{2}, \\
& \frac{2 \pi}{h} \bar{l}_{1}=2 \pi m, \\
& \tilde{n}_{1}, \tilde{n}_{2}, m \in Z .
\end{aligned}
$$

That is,

$$
\begin{aligned}
& \frac{1}{\sqrt{2 E}}=\left(\widetilde{n}_{1}+\widetilde{n}_{2}-m+1\right) h, \\
& \frac{\bar{e}_{1}}{\sqrt{2 E}}=\left(\widetilde{n}_{1}-\widetilde{n}_{2}\right) h, \\
& \bar{l}_{1}=m h .
\end{aligned}
$$

Set $n_{k}=\widetilde{n}_{k}-((m+|m|) / 2), k=1,2$. Then, we obtain

$$
\begin{aligned}
& \frac{1}{\sqrt{2 E}}=\left(n_{1}+n_{2}+|m|+1\right) h, \\
& \frac{\bar{e}_{1}}{\sqrt{2 E}}=\left(n_{1}-n_{2}\right) h,
\end{aligned}
$$




$$
\bar{l}_{1}=m h \text {. }
$$

Substituting these into the classical restriction

$$
\frac{1}{\sqrt{2 E}}>\left|\bar{l}_{1}\right|+\frac{\left|\bar{e}_{1}\right|}{\sqrt{2 E}}
$$

we get $n_{1}+n_{2} \geqq\left|n_{1}-n_{2}\right|$, namely, $n_{1}, n_{2} \geqq 0$. Thus, we have

$$
\begin{aligned}
& E=\frac{1}{2 n^{2} h^{2}}=E_{n}, \quad n=1,2, \cdots, \\
& \bar{e}_{1}=\frac{n_{1}-n_{2}}{n}=\bar{e}_{1, n_{1}, n_{2}}, \\
& \bar{l}_{1}=m h=\bar{l}_{1, m},
\end{aligned}
$$

where

$$
n=n_{1}+n_{2}+|m|+1, \quad n_{1}, n_{2} \geqq 0, \quad n_{1}, n_{2}, m \in \mathbb{Z} .
$$

Hence, we obtain Theorm 1.

For each $n \geqq 1$, it is easy to see that the number of tuples $\left(n_{1}, n_{2}, m\right)$ satisfying $n=n_{1}+n_{2}+|m|+1$ and $n_{1}, n_{2} \geqq 0$ is equal to $n^{2}$. Thus, we get the following. $E_{n}, \bar{e}_{1, n_{1}, n_{2}}, \bar{l}_{1, m}$ are just equal to eigenvalues of $\hat{H}, \hat{e}_{1}$ and $\hat{l}_{1}$, respectively. For each $E_{n}$, the number of $L\left(E_{n}, \bar{l}_{1}, \bar{e}_{1}\right)$ satisfying the quantization condition is equal to the multiplicities of the eigenspace of $\hat{H}$ corresponding to $E_{n}$. (See [5] pp. 119 and 131). Thus, we get Theorem 2.

\section{References}

[1] R. Abraham and J.E. Marsden, Foundations of Mechanics, Benjamin, London, Amsterdam, 1978.

[2] V.I. ARNOLD, Characterstic class entering in quantization conditions, Functional Anal. Appl., 1 (1967), 1-13.

[3] V.I. ARNOLD, Mathematical Methods of Classical Mechanics, Springer-Verlag, New York, Heidlberg, Berlin, 1978.

[4] J.-P. ECKmann and R. SENEOR, The Maslov-WKB method for the (an-) Harmonic oscillator. Arch. Rational Mech. Anal., 61 (1976), 153-173.

[5] L. D. Landau and E. M. Lifshitz, Quantum Mechanics, Pergman Press, Oxford, New York, 1976.

[6] J. LERAY, Analyse lagrangienne et mechanique quantique, Seminaire du College de France 1976-1977; R.C.P. 25, Strasbourg, 1978,

[7] V.P. Maslov, Theorie des Perturbations et Methods Asymptotiques, Dunod, Paris, 1972.

[8] V.P. MASLOV and M. V. FEDORIUK, Semi-classical Approximation in Quantum Mechanics, Reidel, Dordrecht-Boston-London, 1981. 
[9] J.-M. Souriau, Sur le variete de Kepler, Symposia Mathematica, 14, Academic Press, London, 1974, 343-360.

Present Address:

Department of Mathematics

Faculty of Science

TOKYo Metropolitan University

Fukazawa, Setagaya-KU, TOKYo 158 